\title{
Radius of Starlikeness of Certain Analytic Functions
}

\author{
Asha Sebastian and V. Ravichandran
}

\begin{abstract}
This paper studies analytic functions $f$ defined on the open unit disk of the complex plane for which $f / g$ and $(1+z) g / z$ are both functions with positive real part for some analytic function $g$. We determine radius constants of these functions to belong to classes of strong starlike functions, starlike functions of order $\alpha$, parabolic starlike functions, as well as to the classes of starlike functions associated with lemniscate of Bernoulli, cardioid, lune, reverse lemniscate, sine function, exponential function and a particular rational function. The results obtained are sharp.
\end{abstract}

\section{Introduction}

The unit disk $\mathbb{D}$ consists of all points $z \in \mathbb{C}$ satisfying $|z|<1$ and the class $\mathcal{A}$ consists of all analytic functions $f: \mathbb{D} \rightarrow \mathbb{C}$ normalised by the condition $f(0)=f^{\prime}(0)-1=0$. Let $\mathcal{S}$ denote the class of univalent functions in $\mathcal{A}$. For two families $\mathcal{G}$ and $\mathcal{F}$ of $\mathcal{A}$, the $\mathcal{G}$-radius of $\mathcal{F}$ denoted by $R_{\mathcal{G}}(\mathcal{F})$ is the largest number $R$ such that $r^{-1} f(r z) \in \mathcal{G}$ for $0<r \leq R$ and for all $f \in \mathcal{F}$. Whenever $\mathcal{G}$ is characterised by a geometric property $P$, the number $R$ is also referred to as the radius of property $P$ for the class $\mathcal{F}$. There are various studies on radius problems; one of these studies focus on functions $f$ characterised by the ratio between $f$ and another function $g$ belonging to particular subclasses of $\mathcal{A}[\mathbf{3 0}, \mathbf{3 1}, \mathbf{3 2}, \mathbf{3 7}, \mathbf{3 8}, \mathbf{4 1}, 43$. Ali et al. obtained several radius results for classes of functions $f$ satisfying one of the conditions: (i) $\operatorname{Re}(f(z) / g(z))>0$ where $\operatorname{Re}(g(z) / z)>0$ or $\operatorname{Re}(g(z) / z)>1 / 2$ (ii) $|(f(z) / g(z))-1|<1$ where $\operatorname{Re}(g(z) / z)>0$ or $g$ is convex. These classes are closely related to the class of closeto-convex functions and for functions belonging to several related classes we study various radii for these functions to belong to several subclasses of starlike functions discussed below.

An analytic function $f$ is subordinate to another analytic function $g$, written $f \prec g$ or $f(z) \prec g(z)(z \in \mathbb{D})$, if there exists an analytic function $w: \mathbb{D} \rightarrow \mathbb{D}$ with $w(0)=0$ satisfying $f=g \circ w$. For a univalent function $g$, the equivalent condition for subordination is $f(0)=g(0)$ and $f(\mathbb{D}) \subseteq g(\mathbb{D})$. Subclasses of starlike and convex functions are characterised by the quantities $z f^{\prime}(z) / f(z)$ or $1+z f^{\prime \prime}(z) / f^{\prime}(z)$ lying in a convex region in the right half plane. In 1989, Shanmugam [42] unified the classes of starlike and convex functions using subordination and convolution: for a fixed $g \in \mathcal{A}$ and a convex function $\varphi$ with $\varphi(0)=1$, he considered the class $\mathcal{S}_{g}^{*}(\varphi)$ of all functions $f \in \mathcal{A}$ satisfying $z(f * g)^{\prime}(z) /(f * g)(z) \prec \varphi(z)$. Here the function $f * g$ denotes the convolution (or the Hadamard product) of two analytic

2010 Mathematics Subject Classification. 30C80, 30C45.

Key words and phrases. Univalent functions; convex functions; starlike functions; subordination; radius of starlikeness.

The first author is supported by the institute fellowship from NIT Tiruchirappalli. 
functions $f(z)=\sum_{n=1}^{\infty} a_{n} z^{n}$ and $g(z)=\sum_{n=1}^{\infty} b_{n} z^{n}$ defined by $(f * g)(z)=\sum_{n=1}^{\infty} a_{n} b_{n} z^{n}$. When $g(z)=z /(1-z)$, we denote the class $\mathcal{S}_{g}^{*}(\varphi)$ by $\mathcal{S}^{*}(\varphi)$ and when $g(z)=z /(1-z)^{2}$, the class $\mathcal{S}_{g}^{*}(\varphi)$ by $\mathcal{K}(\varphi)$. In 1992, Ma and Minda [29] gave a unified treatment of growth, distortion and covering theorems of functions in the classes $\mathcal{S}^{*}(\varphi)$ and $\mathcal{K}(\varphi)$. Note that $\mathcal{S}^{*}[A, B]:=\mathcal{S}^{*}((1+A z) /(1+B z)), \quad-1 \leq B<A \leq 1$ is the class of Janowski starlike functions and $\mathcal{K}[A, B]:=\mathcal{K}((1+A z) /(1+B z))$ is the class of Janowski convex functions. For $0 \leq \alpha<1$, the classes $\mathcal{S}^{*}(\alpha)=\mathcal{S}^{*}[1-2 \alpha,-1]$ and $\mathcal{K}(\alpha)=\mathcal{K}[1-2 \alpha,-1]$ are the familiar classes of starlike functions of order $\alpha$ and convex functions of order $\alpha$ respectively. These classes were studied extensively in $[\mathbf{8}, \mathbf{1 1}, \mathbf{1 2}, \mathbf{1 3}$. The class $\mathcal{U C V}$ of uniformly convex functions consists of all functions $f \in \mathcal{A}$ that maps every circular arc contained in $\mathbb{D}$ with centre $\xi \in \mathbb{D}$ onto a convex arc. Rønning [40] and Ma and Minda [28] independently proved that a function $f \in \mathcal{U C V}$ if

$$
1+\frac{z f^{\prime \prime}(z)}{f^{\prime}(z)} \prec 1+\frac{2}{\pi^{2}}\left(\log \frac{1+\sqrt{z}}{1-\sqrt{z}}\right)^{2}:=\varphi_{P A R}(z) .
$$

The image $\varphi_{P A R}(\mathbb{D})=\left\{w=u+\mathrm{i} v: v^{2}<2 u-1\right\}=\{w: \operatorname{Re} w>|w-1|\}$ is a parabolic region and the functions in the class $\mathcal{S}_{p}:=\mathcal{S}^{*}\left(\varphi_{P A R}\right)$ are known as parabolic starlike functions. These functions are studied by several authors [1, 10, 28, 39, 43. For $0<\gamma \leq 1$, the class $\mathcal{S}^{*}\left((1+z / 1-z)^{\gamma}\right)$ is the class $\mathcal{S}_{\gamma}^{*}$ of strongly starlike functions of order $\gamma$ and note that the function $f \in \mathcal{S}_{\gamma}^{*}$ if $\left|\arg \left(z f^{\prime}(z) / f(z)\right)\right| \leq \pi \gamma / 2$.

In 1996, Sokół and Stankiewicz [45] and several authors [2, 3, 5, 35] studied the class $\mathcal{S}_{L}^{*}=\mathcal{S}^{*}(\sqrt{1+z})$. Geometrically, the class $\mathcal{S}_{L}^{*}$ represents a collection of functions $f \in \mathcal{A}$ such that $z f^{\prime}(z) / f(z)$ lies in the region bounded by the right half of the lemniscate of Bernoulli $\left|w^{2}-1\right|=1$ or $\left(u^{2}+v^{2}\right)^{2}-2\left(u^{2}-v^{2}\right)=0$. Motivated by these, Mendiratta et al. [33, 34] introduced and studied the sub classes of starlike functions

$$
\mathcal{S}_{e}^{*}=\mathcal{S}^{*}\left(e^{z}\right) \quad \text { and } \quad \mathcal{S}_{R L}^{*}=\mathcal{S}^{*}\left(\sqrt{2}-(\sqrt{2}-1) \sqrt{\frac{1-z}{1+2(\sqrt{2}-1) z}}\right) .
$$

Geometrically, the function $f \in \mathcal{S}_{R L}^{*}$ provided $z f^{\prime}(z) / f(z)$ lies in the interior of the left half of the shifted lemniscate of Bernoulli given by $\left|(w-\sqrt{2})^{2}-1\right|<1$. Similarly, Sharma et al. [44] studied various properties of the class $\mathcal{S}_{c}^{*}=\mathcal{S}^{*}\left(\varphi_{c}(z)\right)$ where $\varphi_{c}(z)=1+(4 / 3) z+$ $(2 / 3) z^{2}$. Essentially, a function $f \in \mathcal{S}_{c}^{*}$ if $z f^{\prime}(z) / f(z)$ lies in the region bounded by the cardioid $\Omega_{c}:=\left\{x+\mathrm{i} y:\left(9 x^{2}+9 y^{2}-18 x+5\right)^{2}-16\left(9 x^{2}+9 y^{2}-6 x+1\right)=0\right\}$. In 2015, Raina and Sokół [36] considered the class $\mathcal{S}_{\mathbb{\zeta}}^{*}=\mathcal{S}^{*}(h)$ where $h(z)=z+\sqrt{1+z^{2}}$. They proved that $f \in \mathcal{S}_{\mathbb{C}}^{*}$ iff $z f^{\prime}(z) / f(z)$ belongs to the lune shaped region $\mathcal{R}:=\left\{w \in \mathbb{C}:\left|w^{2}-1\right|<2|w|\right\}$. Several other properties of $\mathcal{S}_{\mathbb{C}}^{*}$ were discussed by Gandhi and Ravichandran [9]. Kumar and Ravichandran [26] considered the class $\mathcal{S}_{R}^{*}=\mathcal{S}^{*}(\psi)$ where $\psi(z)=1+\left(z k+z^{2} /\left(k^{2}-\right.\right.$ $k z)$ ), $\quad k=\sqrt{2}+1$. Cho et al. [7] in a similar fashion defined the class $\mathcal{S}_{\sin }^{*}=\mathcal{S}^{*}(1+\sin z)$. Recently, several interesting subclasses of starlike functions were studied when $\varphi$ is related to Booth lemniscate [6] and Bell numbers [27.

Kotsur [14] investigated the class of functions $f \in \mathcal{A}$ such that $\operatorname{Re}\left(z f^{\prime}(z)\right)^{\prime} / g^{\prime}(z)>0$ where $g$ is starlike. Similar studies on radii of starlikeness and convexity of certain close to convex functions can be found in $[\mathbf{1 5}, \mathbf{1 6}, \mathbf{1 7}, \mathbf{1 8}$. Kowalczyk and Lecko [19, 20] introduced polynomial close to convex functions and determined radii of selected classes in them. For further related results, see [21, 22, 23. Pranav Kumar and Vasudevarao 25] estimated 
the logarithmic coefficients of certain close to convex functions where $\operatorname{Re}(1-z) f^{\prime}(z)>0$, $\operatorname{Re}\left(1-z^{2}\right) f^{\prime}(z)>0$ and $\operatorname{Re}\left(1-z+z^{2}\right) f^{\prime}(z)>0$. Recently in 2019, Lecko and Sim [24] considered the starlike functions $z /\left(1-z^{2}\right), z /(1-z)^{2}$ and investigated the functions satisfying $\operatorname{Re}\left(1-z^{2}\right) f(z) / z>0$ and $\operatorname{Re}(1-z)^{2} f(z) / z>0$. They have also determined certain sharp coefficient estimates.

Motivated by these studies, we define certain classes of functions $f \in \mathcal{A}$ in the open unit disc $\mathbb{D}$ characterised by its ratio with a certain function $g$. The classes we discuss here consist of functions $f \in \mathcal{A}$ satisfying the following conditions: (i) $\operatorname{Re} f(z) / g(z)>$ 0 where $\operatorname{Re}(1+z) g(z) / z>0$ (ii) $|f(z) / g(z)-1|>0$ where $\operatorname{Re}(1+z) g(z) / z>0$ (iii) $\operatorname{Re}(1+z) f(z) / z>0$ (iv) $\operatorname{Re}(1+z)^{2} f(z) / z>0$. We compute radius constants of the above functions for several interesting subclasses of $\mathcal{A}$ like starlike functions of order $\alpha$, parabolic starlike functions, starlike functions associated with lemniscate of Bernoulli, exponential function, cardioid, sine function, lune, a particular rational function, reverse lemniscate and strong starlike functions. The main technique involved here in finding the radius for the classes of functions is to determine the disk that contains the image of $\mathbb{D}$ by the mapping $z f^{\prime}(z) / f(z)$.

\section{Radius Problems}

Let $\mathcal{P}$ be the class of all analytic functions $p: \mathbb{D} \rightarrow \mathbb{C}$ with $p(0)=1$ and $\operatorname{Re} p(z)>0$ for all $z \in \mathbb{D}$. This class is known as the class of functions with positive real part or the class of Carathéodory functions. This class is used in characterization of several well-studied classes of univalent functions. Our first result concerns the class $\mathcal{F}_{1}$ of functions $f \in \mathcal{A}$ satisfying $f / g \in \mathcal{P}$ for some $g \in \mathcal{A}$ with $(1+z) g(z) / z \in \mathcal{P}$. The functions $f_{1}, g_{1}: \mathbb{D} \longrightarrow \mathbb{C}$ defined by

$$
f_{1}(z)=\frac{z(1-z)^{2}}{(1+z)^{3}} \quad \text { and } \quad g_{1}(z)=\frac{z(1-z)}{(1+z)^{2}}
$$

satisfy

$$
\operatorname{Re} \frac{f_{1}(z)}{g_{1}(z)}=\operatorname{Re} \frac{(1+z) g_{1}(z)}{z}=\operatorname{Re} \frac{1-z}{1+z}>0
$$

and hence the function $f_{1} \in \mathcal{F}_{1}$. This proves that the class $\mathcal{F}_{1}$ is non-empty. This function $f_{1}$ is the extremal function for the radius problems that we consider. As

$$
f_{1}(z)=z-5 z^{2}+13 z^{3}-25 z^{4}+\ldots,
$$

the functions in $\mathcal{F}_{1}$ are not necessarily univalent. Since

$$
f_{1}^{\prime}(z)=\frac{(1-z)(1-5 z)}{(1+z)^{4}}
$$

we have $f_{1}^{\prime}(1 / 5)=0$ and it follows, by the first part of the following theorem, that the radius of univalence of the functions in class $\mathcal{F}_{1}$ is $1 / 5$.

THEOREM 2.1. For the class $\mathcal{F}_{1}$, the following results hold:

(i) The $\mathcal{S}^{*}(\alpha)$ radius $R_{\mathcal{S}^{*}(\alpha)}=2(1-\alpha) /(5+\sqrt{25-(4 \alpha(1-\alpha))}), \quad 0 \leq \alpha<1$.

(ii) The $\mathcal{S}_{L}^{*}$ radius $R_{\mathcal{S}_{L}^{*}}=(2 \sqrt{2}-2) /(5+\sqrt{33-4 \sqrt{2}}) \approx 0.0809$.

(iii) The $\mathcal{S}_{p}$ radius $R_{\mathcal{S}_{p}}=5-2 \sqrt{6} \approx 0.1010$.

(iv) The $\mathcal{S}_{e}^{*}$ radius $R_{\mathcal{S}_{e}^{*}}=(2 e-2) /\left(5 e+\sqrt{25 e^{2}+4(1-e)}\right) \approx 0.1276$. 
(v) The $\mathcal{S}_{c}^{*}$ radius $R_{\mathcal{S}_{c}^{*}}=(15-\sqrt{217}) / 2 \approx 0.1345$.

(vi) The $\mathcal{S}_{\text {sin }}^{*}$ radius $R_{\mathcal{S}_{\text {sin }}^{*}}=2 \sin 1 /(5+\sqrt{25+4 \sin 1(1+\sin 1)}) \approx 0.1589$.

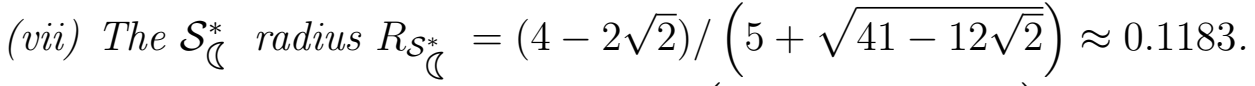

(viii) The $\mathcal{S}_{R}^{*}$ radius $R_{\mathcal{S}_{R}^{*}}=(6-4 \sqrt{2}) /(5+\sqrt{81-40 \sqrt{2}}) \approx 0.0342$.

(ix) The $\mathcal{S}_{R L}^{*}$ radius $R_{\mathcal{S}_{R L}^{*}}$ is the root $(\approx 0.0566)$ in $[0,1]$ of the equation

$$
\begin{aligned}
25 r^{2}+\left(r^{2}-1\right)^{2}+\left(r^{2}-1\right) \sqrt{\left(r^{2}+\sqrt{2}\right)\left(2-\sqrt{2}-r^{2}\right)} & \\
-\left(1+\sqrt{2}\left(r^{2}-1\right)\right)^{2} & =0 .
\end{aligned}
$$

(x) The $\mathcal{S}_{\gamma}^{*}$ radius of strong starlikeness $R_{\mathcal{S}_{\gamma}^{*}} \geq \sin (\pi \gamma / 2) / 5, \quad 0<\gamma \leq 1$.

Proof. Let the function $f \in \mathcal{F}_{1}$. Let the function $g: \mathbb{D} \longrightarrow \mathbb{C}$ be chosen such that

$$
\operatorname{Re} \frac{f(z)}{g(z)}>0 \text { and } \operatorname{Re}\left(\frac{1+z}{z} g(z)\right)>0 \quad(z \in \mathbb{D}) \text {. }
$$

Define the functions $p_{1}, p_{2}: \mathbb{D} \longrightarrow \mathbb{C}$ by

$$
p_{1}(z)=\frac{1+z}{z} g(z) \text { and } \quad p_{2}(z)=\frac{f(z)}{g(z)} .
$$

By (2.2) and (2.3), we have $p_{1}, p_{2} \in \mathcal{P}$ and $f(z)=z p_{1}(z) p_{2}(z) /(1+z)$. Then, a calculation involving logarithmic derivative of the function $f$ shows that

$$
\frac{z f^{\prime}(z)}{f(z)}=\frac{z p_{1}^{\prime}(z)}{p_{1}(z)}+\frac{z p_{2}^{\prime}(z)}{p_{2}(z)}+\frac{1}{1+z} .
$$

The bilinear transformation $1 /(1+z)$ maps the disk $|z| \leq r$ onto the disk

$$
\left|\frac{1}{1+z}-\frac{1}{1-r^{2}}\right| \leq \frac{r}{1-r^{2}} .
$$

For $p \in \mathcal{P}(\alpha):=\{p \in \mathcal{P} \mid \operatorname{Re} p>\alpha\}$, by [41, Lemma 2], we have

$$
\left|\frac{z p^{\prime}(z)}{p(z)}\right| \leq \frac{2(1-\alpha) r}{(1-r)(1+(1-2 \alpha) r)} \quad(|z| \leq r) .
$$

Using (2.5) and (2.6), it follows from (2.4) that the function $f$ maps the disk $|z| \leq r$ onto the disk

$$
\left|\frac{z f^{\prime}(z)}{f(z)}-\frac{1}{1-r^{2}}\right| \leq \frac{5 r}{1-r^{2}}
$$

The classes we discuss here are all subclasses of starlike functions. These classes are described by the quantity $z f^{\prime}(z) / f(z)$ lying in some region in the right half plane. The radius problems are solved by finding $r$ such that the disk in (2.7) is contained in the corresponding regions. By (2.7), we have

$$
\operatorname{Re} \frac{z f^{\prime}(z)}{f(z)} \geq \frac{1-5 r}{1-r^{2}} \geq 0, \quad(r \leq 1 / 5),
$$

then the function $f \in \mathcal{F}_{1}$ is starlike in $|z| \leq 1 / 5$. Hence all the radii that we estimate will be less than $1 / 5$. Note that, for $0<r \leq 1 / 5$, the centre of disk in (2.7) lies in the interval $[1,25 / 24] \approx[1,1.0416]$. 
(i) The number $r=R_{\mathcal{S}^{*}(\alpha)}$ is the root of $\alpha r^{2}-5 r+1-\alpha=0$ in $[0,1]$ and hence, for $0<r \leq R_{\mathcal{S}^{*}(\alpha)}$, it follows from (2.8) that

$$
\operatorname{Re} \frac{z f^{\prime}(z)}{f(z)} \geq \frac{1-5 r}{1-r^{2}} \geq \alpha
$$

For the functions $f_{1} \in \mathcal{F}_{1}$ given by 2.1 we have

$$
\frac{z f_{1}^{\prime}(z)}{f_{1}(z)}=\frac{1-5 z}{1-z^{2}}=\frac{1-5 r}{1-r^{2}}=\alpha, \quad\left(z=r=R_{\mathcal{S}^{*}(\alpha)}\right)
$$

and this shows that the radius is sharp.

(ii) It follows from (2.7) that

$$
\left|\frac{z f^{\prime}(z)}{f(z)}-1\right| \leq\left|\frac{z f^{\prime}(z)}{f(z)}-\frac{1}{1-r^{2}}\right|+\frac{r^{2}}{1-r^{2}} \leq \frac{5 r+r^{2}}{1-r^{2}}
$$

The number $r=R_{\mathcal{S}_{L}^{*}}$ is the root in $[0,1]$ of $\left(5 r+r^{2}\right)=(\sqrt{2}-1)\left(1-r^{2}\right)$ and for $0<r \leq R_{\mathcal{S}_{L}^{*}}$, we have

$$
\frac{5 r+r^{2}}{1-r^{2}} \leq \sqrt{2}-1
$$

Therefore, by 2.9 and 2.10 , for $0<r \leq R_{\mathcal{S}_{L}^{*}}$, we have

$$
\left|\frac{z f^{\prime}(z)}{f(z)}-1\right| \leq \frac{5 r+r^{2}}{1-r^{2}} \leq \sqrt{2}-1
$$

For $0<r \leq R_{\mathcal{S}_{L}^{*}}$, using triangle inequality and 2.11), we have

$$
\left|\frac{z f^{\prime}(z)}{f(z)}+1\right| \leq 2+\left|\frac{z f^{\prime}(z)}{f(z)}-1\right| \leq \sqrt{2}+1
$$

and hence by (2.11) and 2.12),

$$
\left|\left(\frac{z f^{\prime}(z)}{f(z)}\right)^{2}-1\right| \leq\left|\frac{z f^{\prime}(z)}{f(z)}+1\right|\left|\frac{z f^{\prime}(z)}{f(z)}-1\right| \leq(\sqrt{2}+1)(\sqrt{2}-1)=1 .
$$

The number $\rho=R_{\mathcal{S}_{L}^{*}}$ satisfies $(1+5 \rho) /\left(1-\rho^{2}\right)=\sqrt{2}$. Using this, we see that the function $f_{1}$ defined in 2.1 satisfies

$$
\left|\left(\frac{z f_{1}^{\prime}(z)}{f_{1}(z)}\right)^{2}-1\right|=\left|\left(\frac{1-5 z}{1-z^{2}}\right)^{2}-1\right|=\left|\left(\frac{1+5 \rho}{1-\rho^{2}}\right)^{2}-1\right|=1, \quad\left(z:=-\rho=-R_{\mathcal{S}_{L}^{*}}\right) .
$$

This shows that the radius is sharp (See Figure 1. (a)).

(iii) Let $\Omega_{P A R}=\left\{w=u+\mathrm{i} v: v^{2}<2 u-1\right\}=\{w: \operatorname{Re} w>|w-1|\}$. Note that $\Omega_{P A R}$ is the interior of a parabola in the right half plane which is symmetric about real axis and has vertex at $(1 / 2,0)$. By Lemma [43, pp.321], for $1 / 2<a<3 / 2$, we have

$$
\{w \in \mathbb{C}:|w-a|<a-1 / 2\} \subseteq \Omega_{P A R} .
$$

If $0<r \leq R_{\mathcal{S}_{p}}$, then $a=1 /\left(1-r^{2}\right) \leq 3 / 2$ and

$$
\frac{5 r}{1-r^{2}} \leq \frac{1}{1-r^{2}}-\frac{1}{2} \text {. }
$$




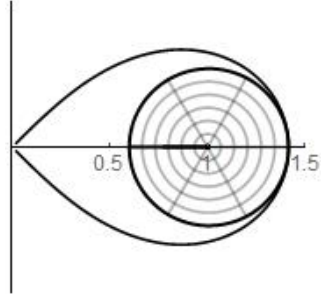

(A) Sharpness of class $\mathcal{S}_{L}^{*}$

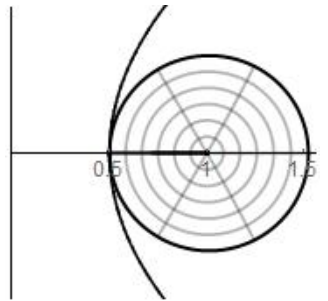

(B) Sharpness of class $\mathcal{S}_{p}$

FiguRE 1. Sharpness of starlike functions associated with lemniscate and parabolic starlike functions.

Thus, by (2.13), we see that the disk in (2.7) lies inside the parabolic region $\Omega_{P A R}$. Sharpness follows for the function $f_{1}$ defined in (2.1) (See Figure 1. (b)). At $z:=\rho=R_{\mathcal{S}_{p}}$, we have

$$
\operatorname{Re} \frac{z f^{\prime}(z)}{f(z)}=\frac{1-5 \rho}{1-\rho^{2}}=\frac{5 \rho-\rho^{2}}{1-\rho^{2}}=\left|\frac{\rho^{2}-5 \rho}{1-\rho^{2}}\right|=\left|\frac{z f^{\prime}(z)}{f(z)}-1\right| .
$$

(iv) For $e^{-1} \leq a \leq\left(e+e^{-1}\right) / 2$, by [34, Lemma 2.2], we have

$$
\left\{w \in \mathbb{C}:|w-a|<a-e^{-1}\right\} \subseteq\{w \in \mathbb{C}:|\log w|<1\}=: \Omega_{e} .
$$

For $0<r \leq R_{\mathcal{S}_{e}^{*}}$, we have $1 / e \leq a=1 /\left(1-r^{2}\right) \leq\left(e+e^{-1}\right) / 2$ and

$$
\frac{5 r}{1-r^{2}} \leq \frac{1}{1-r^{2}}-\frac{1}{e}
$$

By (2.14), the disk in (2.7) lies inside $\Omega_{e}$ for $0<r \leq R_{\mathcal{S}_{e}^{*}}$ proving that the $\mathcal{S}_{e}^{*}$ radius for the class $\mathcal{F}_{1}$ is $R_{\mathcal{S}_{e}^{*}}$. The sharpness follows for the function $f_{1}$ defined in (2.1) (See Figure 2.(a). Indeed at $z:=\rho=R_{\mathcal{S}_{e}^{*}}$, we have

$$
\left|\log \frac{z f_{1}^{\prime}(z)}{f_{1}(z)}\right|=\left|\log \frac{1-5 \rho}{1-\rho^{2}}\right|=1 .
$$

(v) For $1 / 3<a \leq 5 / 3$, by [44, Lemma 2.5], we have

$$
\{w \in \mathbb{C}:|w-a|<(3 a-1) / 3\} \subseteq \Omega_{c}
$$

where $\Omega_{c}$ is the region bounded by the cardioid $\left\{x+i y:\left(9 x^{2}+9 y^{2}-18 x+5\right)^{2}-16\left(9 x^{2}+\right.\right.$ $\left.\left.9 y^{2}-6 x+1\right)=0\right\}$. If $0<r \leq R_{\mathcal{S}_{c}^{*}}$, then

$$
\frac{5 r}{1-r^{2}} \leq \frac{1}{1-r^{2}}-\frac{1}{3}
$$

By 2.15), we see that the disk in 2.7) lies inside $\Omega_{c}$, if $0<r \leq R_{\mathcal{S}_{c}^{*}}$. The result is sharp for the function $f_{1}$ defined in 2.1 . At $z:=\rho=R_{\mathcal{S}_{c}^{*}}$,

$$
\left|\frac{z f^{\prime}(z)}{f(z)}\right|=\left|\frac{1-5 \rho}{1-\rho^{2}}\right|=\frac{1}{3}=\Omega_{c}(-1) \in \partial_{c}(\mathbb{D}) .
$$

(vi) For $|a-1| \leq \sin 1$, by [7, Lemma 3.3], we have

$$
\{w \in \mathbb{C}:|w-a|<\sin 1-|a-1|\} \subseteq \Omega_{s}
$$




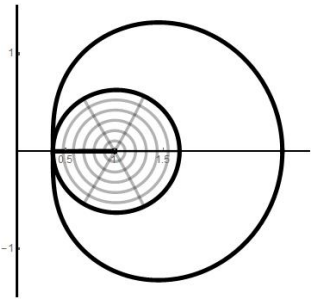

(A) Sharpness of class $\mathcal{S}_{e}^{*}$

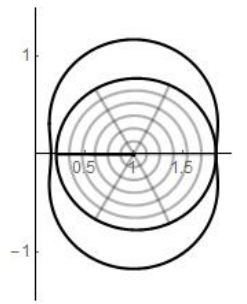

(B) Sharpness of class $\mathcal{S}_{\text {sin }}^{*}$

FiguRE 2. Sharpness for starlike functions associated with exponential and sine functions.

where $\Omega_{s}:=q_{0}(\mathbb{D})$ is the image of the unit disk $\mathbb{D}$ under the mappings $q_{0}(z)=1+\sin z$. It is evident from (2.7) and (2.16) that

$$
\frac{5 r}{1-r^{2}} \leq \sin 1-\frac{r^{2}}{1-r^{2}}
$$

Hence the disk in (2.7) lies inside $\Omega_{s}$ provided $0<r \leq R_{\mathcal{S}_{\text {sin }}^{*}}$. For the function $f_{1}$ defined in (2.1) (See Figure 2(b)), at $z:=-\rho=-R_{\mathcal{S}_{\text {sin }}^{*}}$,

$$
\left|\frac{z f^{\prime}(z)}{f(z)}\right|=\left|\frac{1+5 \rho}{1-\rho^{2}}\right|=1+\sin 1=q_{0}(1) \in \partial \Omega_{s}(\mathbb{D}) .
$$

(vii) Let $\mathcal{S}_{\overparen{C}}^{*}=\left\{f \in \mathcal{S}^{*}:\left|\left(z f^{\prime}(z) / f(z)\right)^{2}-1\right|<2\left|z f^{\prime}(z) / f(z)\right|\right\}$. In 2015, Sokół [36] proved that if $f \in \mathcal{S}_{\mathfrak{\zeta}}^{*}$ then the function $f$ is a starlike function and $\left|z f^{\prime}(z) / f(z)-1\right|<\sqrt{2}$ and $\left|z f^{\prime}(z) / f(z)+1\right|>\sqrt{2}$. Interpreting these conditions geometrically, for $f \in \mathcal{S}_{\zeta}^{*}$ we see that $w=z f^{\prime}(z) / f(z)$ lies in the right half plane, inside the intersection of disks $\{w:|w-1|<\sqrt{2}\}$ and $\{w:|w+1|<\sqrt{2}\}$. According to [9, Lemma 2.1], we have

$$
\{w \in \mathbb{C}:|w-a|<1-|\sqrt{2}-a|\} \subseteq\left\{w \in \mathbb{C}:\left|w^{2}-1\right|<2|w|\right\}
$$

or, $(1-\sqrt{2}) r^{2}+5 r+\sqrt{2}-2 \leq 0$. If $0<r \leq R_{\mathcal{S}_{\mathbb{g}}^{*}}$, then

$$
\frac{5 r-1}{1-r^{2}} \leq 1-\sqrt{2}
$$

Thus, by (2.17), the disk in (2.7) lies inside $\left\{w \in \mathbb{C}:\left|w^{2}-1\right|<2|w|\right\}$ and hence $f \in \mathcal{S}_{\overparen{\zeta}}^{*}$. The sharpness follows from the functions defined in 2.1. At $z:=\rho=R_{\mathcal{S}_{\mathbb{\zeta}}^{*}}$, we have

$$
\left|\left(\frac{z f_{1}^{\prime}(z)}{f_{1}(z)}\right)^{2}-1\right|=\left|\left(\frac{1-5 \rho}{1-\rho^{2}}\right)^{2}-1\right|=2\left|\frac{1-5 \rho}{1-\rho^{2}}\right|=2\left|\frac{z f_{1}^{\prime}(z)}{f_{1}(z)}\right| .
$$

(viii) For $2(\sqrt{2}-1)<a \leq \sqrt{2}$, by [26, Lemma 2.2],

$$
\{w \in \mathbb{C}:|w-a|<a-2(\sqrt{2}-1)\} \subseteq \psi(\mathbb{D})
$$

where $\psi$ is given by $\psi(z)=1+\left(z^{2} k+z^{2} /\left(k^{2}-k z\right)\right), \quad k=\sqrt{2}+1$. If $0<r \leq R_{\mathcal{S}_{R}^{*}}$, $2(\sqrt{2}-1)<a=1 /\left(1-r^{2}\right) \leq \sqrt{2}$ and

$$
\frac{5 r-1}{1-r^{2}} \leq 2-2 \sqrt{2}, \quad\left(0<r \leq R_{\mathcal{S}_{R}^{*}}\right) .
$$


Then, by (2.18), the disk in (2.7) lies inside $\psi(\mathbb{D})$. The result is sharp for the function defined in (2.1). At $z:=\rho=R_{\mathcal{S}_{R}^{*}}$,

$$
\left|\frac{z f^{\prime}(z)}{f(z)}\right|=\left|\frac{1-5 \rho}{1-\rho^{2}}\right|=2(\sqrt{2}-1)=\psi(1) \in \partial \psi(\mathbb{D}) .
$$

(ix) For $\sqrt{2} / 3 \leq a<\sqrt{2}$, by [33, Lemma 3.2], we have

$$
\left\{w \in \mathbb{C}:|w-a|<r_{R L}\right\} \subseteq\left\{w \in \mathbb{C}:\left|(w-\sqrt{2})^{2}-1\right|<1\right\},
$$

provided $r_{R L}=\left(\left(1-(\sqrt{2}-a)^{2}\right)^{1 / 2}-\left(1-(\sqrt{2}-a)^{2}\right)\right)^{1 / 2}$. If $0<r \leq R_{\mathcal{S}_{R L}^{*}}$, then it follows that $\sqrt{2} / 3 \leq a=1 /\left(1-r^{2}\right)<\sqrt{2}$, and

$$
\begin{aligned}
25 r^{2}-\left(1-r^{2}\right) & \sqrt{\left(1-r^{2}\right)^{2}-\left(\left(\sqrt{2}-\sqrt{2} r^{2}\right)-1\right)^{2}} \\
& +\left(1-r^{2}\right)^{2}-\left(\left(\sqrt{2}-\sqrt{2} r^{2}\right)-1\right)^{2} \leq 0 .
\end{aligned}
$$

Then, by (2.19), the disk in (2.7) lies inside the region $\left\{w:\left|(w-\sqrt{2})^{2}-1\right|<1\right\}$. The result is sharp for the function defined in (2.1).

(x) If a function $f(z)$ is strongly starlike of order $\gamma, 0<\gamma \leq 1$, then $\left|\arg \left\{z f^{\prime}(z) / f(z)\right\}\right| \leq$ $\pi \gamma / 2$. In other words the values of $\left|z f^{\prime}(z) / f(z)\right|$ are in the sector $|y| \leq \tan (\pi \gamma / 2) x$, $x \geq 0$. In 1997, Gangadharan et al. [10, Lemma 3.1] proved that

$$
\{w \in \mathbb{C}:|w-a|<a \sin (\pi \gamma / 2)\} \subseteq\{w:|\arg w| \leq(\pi \gamma) / 2\}, \quad 0<\gamma \leq 1 .
$$

If $0<r \leq R_{\mathcal{S}_{\gamma}^{*}}, 0<\gamma \leq 1$, then $r \leq(\sin (\pi \gamma) / 2) / 5$. It is evident from 2.20 that the disk in (2.7) is contained in the sector $|\arg w| \leq(\pi \gamma) / 2,0<\gamma \leq 1$ if $0<r \leq R_{\mathcal{S}_{\gamma}^{*}}$.

REMARK 2.2. (i) The class

$$
\mathcal{S}^{*}(c, d)=\left\{f \in \mathcal{A}:\left|\frac{z f^{\prime}(z)}{f(z)}-c\right|<d\right\}
$$

is very closely related to the class of Janowski starlike functions. For the class $\mathcal{F}_{1}$, the $\mathcal{S}_{p}$ radius, $\mathcal{S}^{*}(1 / 2)$ radius and $\mathcal{S}^{*}(1,1 / 2)$ radius are all equal. It is also clear that $\mathcal{S}^{*}(1, \sqrt{2}-$ $1) \subseteq \mathcal{S}_{L}^{*}$. The $\mathcal{S}_{L}^{*}$ radius and $\mathcal{S}^{*}(1, \sqrt{2}-1)$ radius are also equal.

(ii) The radius of strong starlikeness is clearly sharp for $\gamma=0$ and for other cases, we have given a lower bound only.

Definition 2.3. Let $\mathcal{F}_{2}$ be the class of functions $f \in \mathcal{A}$ satisfying the inequality

$$
\left|\frac{f(z)}{g(z)}-1\right|<1 \quad(z \in \mathbb{D})
$$

for some $g \in \mathcal{A}$ with

$$
\operatorname{Re}\left(\frac{1+z}{z} g(z)\right)>0 \quad(z \in \mathbb{D}) .
$$

The functions $f_{2}, g_{2}: \mathbb{D} \longrightarrow \mathbb{C}$ defined by

$$
f_{2}(z)=\frac{z(1-z)^{2}}{(1+z)^{2}} \quad \text { and } \quad g_{2}(z)=\frac{z(1-z)}{(1+z)^{2}}
$$


satisfy

$$
\left|\frac{f_{2}(z)}{g_{2}(z)}-1\right|=|z|<1, \quad \operatorname{Re} \frac{(1+z)}{z} g_{2}(z)=\operatorname{Re} \frac{1-z}{1+z}>0
$$

and hence the function $f_{2} \in \mathcal{F}_{2}$. This proves that the class $\mathcal{F}_{2}$ is non-empty and the function $f_{2}$ is extremal function for the radius problems we consider. As

$$
f_{2}(z)=z-4 z^{2}+8 z^{3}-12 z^{4}+\ldots,
$$

the functions in $\mathcal{F}_{2}$ are not necessarily univalent. Since

$$
f_{2}^{\prime}(z)=\frac{1-5 z+3 z^{2}+z^{3}}{(1+z)^{3}}
$$

we have $f_{2}^{\prime}(\sqrt{5}-2)=0$ and it follows by the first part of the following theorem, that the radius of univalence of the functions in class $\mathcal{F}_{2}$ is $\sqrt{5}-2 \approx 0.2361$.

TheOREM 2.4. For the class $\mathcal{F}_{2}$ the following results hold:

(i) The $\mathcal{S}^{*}(\alpha)$ radius $R_{\mathcal{S}^{*}(\alpha)}=(1-\alpha) /\left(2+\sqrt{4+(\alpha-1)^{2}}\right), \quad 0 \leq \alpha<1$.

(ii) The $\mathcal{S}_{L}^{*}$ radius $R_{\mathcal{S}_{L}^{*}} \geq(\sqrt{5}-2) /(1+\sqrt{2}) \approx 0.0977$.

(iii) The $\mathcal{S}_{p}$ radius $R_{\mathcal{S}_{p}}=\sqrt{17}-4 \approx 0.1231$.

(iv) The $\mathcal{S}_{e}^{*}$ radius $R_{\mathcal{S}_{e}^{*}}=(2 e-2) /\left(4 e+\sqrt{20 e^{2}-8 e+4}\right) \approx 0.1543$.

(v) The $\mathcal{S}_{c}^{*}$ radius $R_{\mathcal{S}_{c}^{*}}=\sqrt{10}-3 \approx 0.1623$.

(vi) The $\mathcal{S}_{\text {sin }}^{*}$ radius $R_{\mathcal{S}_{\text {sin }}^{*}} \geq \sin 1 /(2+\sqrt{4+\sin 1(2+\sin 1)}) \approx 0.1858$.

(vii) The $\mathcal{S}_{\mathbb{\zeta}}^{*}$ radius $R_{\mathcal{S}_{\mathbb{\zeta}}^{*}}=(2-\sqrt{2}) /(2+\sqrt{10-4 \sqrt{2}}) \approx 0.1434$.

(viii) The $\mathcal{S}_{R}^{*}$ radius $R_{\mathcal{S}_{R}^{*}}=(3-2 \sqrt{2}) /(2+\sqrt{21-12 \sqrt{2}}) \approx 0.0428$.

(ix) The $\mathcal{S}_{R L}^{*}$ radius $R_{\mathcal{S}_{R L}^{*}}$ is atleast the smallest root $(\approx 0.0692)$ in $[0,1]$ of the equation

$$
\begin{aligned}
r^{2}(r+4)^{2}+\left(r^{2}-1\right)^{2}+\left(r^{2}-1\right) \sqrt{\left(r^{2}+\sqrt{2}\right)\left(2-\sqrt{2}-r^{2}\right)} & \\
-\left(1+\sqrt{2}\left(r^{2}-1\right)\right)^{2} & =0 .
\end{aligned}
$$

(x) The $\mathcal{S}_{\gamma}^{*}$ radius $R_{\mathcal{S}_{\gamma}^{*}} \geq \sin (\pi \gamma / 2) /(2+\sqrt{4+\sin (\pi \gamma / 2)}, \quad 0<\gamma \leq 1$.

Proof. Let the function $f \in \mathcal{F}_{2}$. Then $|f(z) / g(z)-1|<1$ if and only if $\operatorname{Re}(g(z) / f(z))>$ $1 / 2$. Let the function $g: \mathbb{D} \longrightarrow \mathbb{C}$ be chosen such that

$$
\operatorname{Re} \frac{g(z)}{f(z)}>1 / 2 \text { and } \operatorname{Re}\left(\frac{1+z}{z} g(z)\right)>0 \quad(z \in \mathbb{D}) \text {. }
$$

Define the functions $p_{1}, p_{2}: \mathbb{D} \longrightarrow \mathbb{C}$ by

$$
p_{1}(z)=\frac{1+z}{z} g(z) \text { and } \quad p_{2}(z)=\frac{g(z)}{f(z)} .
$$

By 2.22 and 2.23), we have $p_{1} \in \mathcal{P}, p_{2} \in \mathcal{P}(1 / 2)$ and $f(z)=z p_{1}(z) /\left((1+z) p_{2}(z)\right)$. It can be shown by calculation that

$$
\frac{z f^{\prime}(z)}{f(z)}=\frac{z p_{1}^{\prime}(z)}{p_{1}(z)}-\frac{z p_{2}^{\prime}(z)}{p_{2}(z)}+\frac{1}{1+z} .
$$


Using (2.5) and (2.6), it follows from (2.24) that the function $f$ maps the disk $|z| \leq r$ onto the disk

$$
\left|\frac{z f^{\prime}(z)}{f(z)}-\frac{1}{1-r^{2}}\right| \leq \frac{4 r+r^{2}}{1-r^{2}}
$$

The classes we discuss here are all subclasses of starlike functions. By (2.25), we have

$$
\operatorname{Re} \frac{z f^{\prime}(z)}{f(z)} \geq \frac{1-4 r-r^{2}}{1-r^{2}} \geq 0, \quad(r \leq \sqrt{5}-2) .
$$

Hence all the radii that we estimate will be less than $\sqrt{5}-2 \approx 0.2361$. Also, for $0<r \leq$ $\sqrt{5}-2$, the centre of disk in 2.25 lies in the interval $[1,1 / 4(\sqrt{5}-2)] \approx[1,1.05902]$.

(i) The number $r=R_{\mathcal{S}^{*}(\alpha)}$ is the root of $(\alpha-1) r^{2}-4 r+1-\alpha=0$ in $[0,1]$ and hence, for $0<r \leq R_{\mathcal{S}^{*}(\alpha)}$, it follows by 2.26 that

$$
\operatorname{Re} \frac{z f^{\prime}(z)}{f(z)} \geq \frac{1-4 r-r^{2}}{1-r^{2}} \geq \alpha .
$$

For the functions $f_{2} \in \mathcal{F}_{2}$ given by (2.21), we have

$$
\frac{z f_{2}^{\prime}(z)}{f_{2}(z)}=\frac{1-4 z-z^{2}}{1-z^{2}}=\frac{1-4 r-r^{2}}{1-r^{2}}=\alpha, \quad\left(z=r=R_{\mathcal{S}^{*}(\alpha)}\right)
$$

and this shows that the radius is sharp.

(ii) It follows from (2.25) that

$$
\left|\frac{z f^{\prime}(z)}{f(z)}-1\right| \leq\left|\frac{z f^{\prime}(z)}{f(z)}-\frac{1}{1-r^{2}}\right|+\frac{r^{2}}{1-r^{2}} \leq \frac{4 r+2 r^{2}}{1-r^{2}} .
$$

The number $r=R_{\mathcal{S}_{L}^{*}}$ is the root in $[0,1]$ of $\left(4 r+2 r^{2}\right)=\sqrt{2}-1\left(1-r^{2}\right)$ and for $0<r \leq R_{\mathcal{S}_{L}^{*}}$, we have

$$
\frac{4 r+2 r^{2}}{1-r^{2}} \leq \sqrt{2}-1
$$

Therefore, by (2.27) and $(2.28)$, for $0<r \leq R_{\mathcal{S}_{L}^{*}}$, we have

$$
\left|\frac{z f^{\prime}(z)}{f(z)}-1\right| \leq \frac{4 r+2 r^{2}}{1-r^{2}} \leq \sqrt{2}-1 .
$$

For $0<r \leq R_{\mathcal{S}_{L}^{*}}$, using triangle inequality and 2.29 , we have

$$
\left|\frac{z f^{\prime}(z)}{f(z)}+1\right| \leq \sqrt{2}+1
$$

and hence by (2.29) and (2.30)

$$
\left|\left(\frac{z f^{\prime}(z)}{f(z)}\right)^{2}-1\right| \leq\left|\frac{z f^{\prime}(z)}{f(z)}+1\right|\left|\frac{z f^{\prime}(z)}{f(z)}-1\right| \leq(\sqrt{2}+1)(\sqrt{2}-1)=1 .
$$

The result obtained is not sharp.

(iii) If $0<r \leq R_{\mathcal{S}_{p}}$, then $a=1 /\left(1-r^{2}\right) \leq 3 / 2$ and

$$
\frac{4 r+r^{2}}{1-r^{2}} \leq \frac{1}{1-r^{2}}-\frac{1}{2} \text {. }
$$


Thus, by (2.13), we see that the disk in (2.25) lies inside the parabolic region $\Omega_{P A R}$. Sharpness follows for the function $f_{2}$ defined in (2.21). At $z:=\rho=R_{\mathcal{S}_{p}}$, we have

$$
\operatorname{Re} \frac{z f^{\prime}(z)}{f(z)}=\frac{1-4 \rho-\rho^{2}}{1-\rho^{2}}=\frac{4 \rho}{1-\rho^{2}}=\left|\frac{-4 \rho}{1-\rho^{2}}\right|=\left|\frac{z f^{\prime}(z)}{f(z)}-1\right| .
$$

(iv) For $0<r \leq R_{\mathcal{S}_{e}^{*}}$, we have $1 / e \leq a=1 /\left(1-r^{2}\right) \leq\left(e+e^{-1}\right) / 2$ and

$$
\frac{4 r+r^{2}}{1-r^{2}} \leq \frac{1}{1-r^{2}}-\frac{1}{e}
$$

By (2.14), the disk in 2.25) lies inside $\Omega_{e}$ for $0<r \leq R_{\mathcal{S}_{e}^{*}}$ proving that the $\mathcal{S}_{e}^{*}$ radius for the class $\mathcal{F}_{2}$ is $R_{\mathcal{S}_{e}^{*}}$. The sharpness follows for the function $f_{2}$ defined in (2.21). Indeed at $z:=\rho=R_{\mathcal{S}_{e}^{*}}$, we have

$$
\left|\log \frac{z f_{2}^{\prime}(z)}{f_{2}(z)}\right|=\left|\log \frac{1-4 \rho-\rho^{2}}{1-\rho^{2}}\right|=1 .
$$

(v) If $0<r \leq R_{\mathcal{S}_{c}^{*}}$, then

$$
\frac{4 r+r^{2}}{1-r^{2}} \leq \frac{1}{1-r^{2}}-\frac{1}{3} .
$$

By (2.15), we see that the disk in (2.25) lies inside $\Omega_{c}$, if $0<r \leq R_{\mathcal{S}_{c}^{*}}$. The result is sharp for the function $f_{2}$ defined in (2.21) (See Figure 3. (a)). At $z:=\rho=R_{\mathcal{S}_{c}^{*}}$,

$$
\left|\frac{z f^{\prime}(z)}{f(z)}\right|=\left|\frac{1-4 \rho-\rho^{2}}{1-\rho^{2}}\right|=\frac{1}{3}=\Omega_{c}(-1) \in \partial \Omega_{c}(\mathbb{D}) .
$$

(vi) For $0<r \leq R_{\mathcal{S}_{s i n}^{*}},|a-1| \leq \sin 1$ and

$$
\frac{4 r+r^{2}}{1-r^{2}} \leq \sin 1-\frac{r^{2}}{1-r^{2}},
$$

then it is evident from (2.16) that the disk in 2.25) lies inside $\Omega_{s}$. The radius is not sharp.

(vii) If $0<r \leq R_{\mathcal{S}_{\mathbb{Q}}^{*}}$, then

$$
\frac{r^{2}+4 r-1}{1-r^{2}} \leq 1-\sqrt{2} .
$$

Thus by (2.17), the disk in (2.25) lies inside $\left\{w \in \mathbb{C}:\left|w^{2}-1\right|<2|w|\right\}$ and hence $f \in \mathcal{S}_{\mathbb{C}}^{*}$. The sharpness follows for the functions defined in (2.21) (See Figure 3.(b)). At $z:=\rho=R_{\mathcal{S}_{\mathbb{S}}^{*}}$, we have

$$
\left|\left(\frac{z f_{2}^{\prime}(z)}{f_{2}(z)}\right)^{2}-1\right|=\left|\left(\frac{\rho^{2}+4 \rho-1}{1-\rho^{2}}\right)^{2}-1\right|=2\left|\frac{\rho^{2}+4 \rho-1}{1-\rho^{2}}\right|=2\left|\frac{z f_{1}^{\prime}(z)}{f_{1}(z)}\right| .
$$

(viii) If $0<r \leq R_{\mathcal{S}_{R}^{*}}, 2(\sqrt{2}-1)<a=1 /\left(1-r^{2}\right) \leq \sqrt{2}$ and

$$
\frac{r^{2}+4 r-1}{1-r^{2}} \leq 2-2 \sqrt{2}, \quad 0<r \leq R_{\mathcal{S}_{R}^{*}}
$$




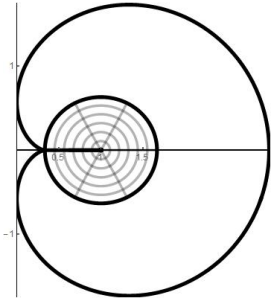

(A) Sharpness of class $\mathcal{S}_{c}^{*}$

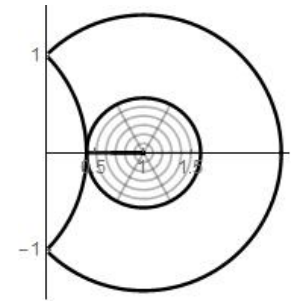

(B) Sharpness of class $\mathcal{S}_{\overparen{乃}}^{*}$

FIGURE 3. Sharpness of starlike functions associated with cardioid and lune.

Then, by (2.18), the disk in 2.25 lies inside $\psi(\mathbb{D})$. For the function defined in 2.21, at $z:=\rho=R_{\mathcal{S}_{R}^{*}}$,

$$
\left|\frac{z f^{\prime}(z)}{f(z)}\right|=\left|\frac{1-4 \rho-\rho^{2}}{1-\rho^{2}}\right|=2(\sqrt{2}-1)=\psi(1) \in \partial \psi(\mathbb{D}) .
$$

(ix) If $0<r \leq R_{\mathcal{S}_{R L}^{*}}, \sqrt{2} / 3 \leq a=1 /\left(1-r^{2}\right)<\sqrt{2}$, and

$$
\begin{aligned}
\left(4 r+r^{2}\right)^{2}-\left(1-r^{2}\right) & \sqrt{\left(1-r^{2}\right)^{2}-\left(\left(\sqrt{2}-\sqrt{2} r^{2}\right)-1\right)^{2}} \\
& +\left(1-r^{2}\right)^{2}-\left(\left(\sqrt{2}-\sqrt{2} r^{2}\right)-1\right)^{2} \leq 0 .
\end{aligned}
$$

Then by 2.19) the disk in 2.25) lies inside the region $\left\{w:\left|(w-\sqrt{2})^{2}-1\right|<1\right\}$. The result obtained is not sharp.

(x) If $0<r \leq R_{\mathcal{S}_{\gamma}^{*}}$ and $0<\gamma \leq 1$, then $r^{2}+4 r \leq(\sin (\pi \gamma) / 2)$. It is evident from (2.20) that the disk in 2.25) is contained in the sector $|\arg w| \leq(\pi \gamma) / 2,0<\gamma \leq 1$ if $0<r \leq R_{\mathcal{S}_{\gamma}^{*}}$.

The problem of determination of the exact radii of starlikeness associated with lemniscate of Bernoulli, sine function, reverse lemniscate and strongly starlike function is open.

Definition 2.5. Let $\mathcal{F}_{3}$ be the class of functions $f \in \mathcal{A}$ satisfying the inequality

$$
\operatorname{Re}\left(\frac{1+z}{z} f(z)\right)>0 \quad(z \in \mathbb{D}) \text {. }
$$

The function $f_{3}: \mathbb{D} \longrightarrow \mathbb{C}$ defined by

$$
f_{3}(z)=\frac{z(1-z)}{(1+z)^{2}}
$$

satisfy

$$
\operatorname{Re} \frac{(1+z) f_{3}(z)}{z}=\operatorname{Re} \frac{1-z}{1+z}>0
$$

and hence the function $f_{3} \in \mathcal{F}_{3}$. This proves that the class $\mathcal{F}_{3}$ is non-empty. This function $f_{3}$ is extremal function for the radii problem we consider. As

$$
f_{3}(z)=z-3 z^{2}+5 z^{3}-7 z^{4}+\ldots
$$

the functions in $\mathcal{F}_{3}$ are not necessarily univalent. Since

$$
f_{3}^{\prime}(z)=\frac{1-3 z}{(1+z)^{3}}
$$


we have $f_{3}^{\prime}(1 / 3)=0$ and it follows, by the first part of the following theorem, that the radius of univalence of the functions in class $\mathcal{F}_{3}$ is $1 / 3 \approx 0.3333$.

THEOREM 2.6. For the class $\mathcal{F}_{3}$, the following results hold:

(i) The $\mathcal{S}^{*}(\alpha)$ radius $R_{\mathcal{S}^{*}(\alpha)}=2(1-\alpha) /(3+\sqrt{9-4 \alpha(1-\alpha)}), \quad 0 \leq \alpha<1$.

(ii) The $\mathcal{S}_{L}^{*}$ radius $R_{\mathcal{S}_{L}^{*}}=(2 \sqrt{2}-2) /(3+\sqrt{9-4 \sqrt{2}(1-\sqrt{2})}) \approx 0.1301$.

(iii) The $\mathcal{S}_{p}$ radius $R_{\mathcal{S}_{p}}=3-2 \sqrt{2} \approx 0.1716$.

(iv) The $\mathcal{S}_{e}^{*}$ radius $R_{\mathcal{S}_{e}^{*}}=(2 e-2) /\left(3 e+\sqrt{9 e^{2}+4(1-e)}\right) \approx 0.2165$.

(v) The $\mathcal{S}_{c}^{*}$ radius $R_{\mathcal{S}_{c}^{*}}=(9-\sqrt{73}) / 2 \approx 0.2279$.

(vi) The $\mathcal{S}_{\text {sin }}^{*}$ radius $R_{\mathcal{S}_{\text {sin }}^{*}}=2 \sin 1 /(3+\sqrt{9+4 \sin 1(1+\sin 1)}) \approx 0.2439$.

(vii) The $\mathcal{S}_{\mathbb{Q}}^{*}$ radius $R_{\mathcal{S}_{\mathbb{R}}^{*}}=(4-2 \sqrt{2}) /(3+\sqrt{25-12 \sqrt{2}}) \approx 0.2008$.

(viii) The $\mathcal{S}_{R}^{*}$ radius $R_{\mathcal{S}_{R}^{*}}=(6-4 \sqrt{2}) /(3+\sqrt{65-40 \sqrt{2}}) \approx 0.0581$.

(ix) The $\mathcal{S}_{R L}^{*}$ radius $R_{\mathcal{S}_{R L}^{*}}$ is the root $(\approx 0.0926)$ in $[0,1]$ of the equation

$$
\begin{aligned}
9 r^{2}+\left(r^{2}-1\right)^{2}+\left(r^{2}-1\right) \sqrt{\left(r^{2}+\sqrt{2}\right)\left(2-\sqrt{2}-r^{2}\right)} & \\
-\left(1+\sqrt{2}\left(r^{2}-1\right)\right)^{2} & =0 .
\end{aligned}
$$

(x) The $\mathcal{S}_{\gamma}^{*}$ radius $R_{\mathcal{S}_{\gamma}^{*}} \geq \sin (\pi \gamma / 2) / 3, \quad 0<\gamma \leq 1$.

Proof. Let the function $f \in \mathcal{F}_{3}$. Then

$$
\operatorname{Re}\left(\frac{1+z}{z} f(z)\right)>0 \quad(z \in \mathbb{D}) .
$$

Define the function $h: \mathbb{D} \longrightarrow \mathbb{C}$ by

$$
h(z)=\frac{1+z}{z} f(z) .
$$

By (2.32) and (2.33) we have $h \in \mathcal{P}$ and $f(z)=z h(z) /(1+z)$.

Therefore, by calculation it can be shown that

$$
\frac{z f^{\prime}(z)}{f(z)}=\frac{z h^{\prime}(z)}{h(z)}+\frac{1}{1+z} .
$$

Using (2.5) and (2.6), it follows from (2.34) that $f$ maps the disk $|z| \leq r$ onto the disk

$$
\left|\frac{z f^{\prime}(z)}{f(z)}-\frac{1}{1-r^{2}}\right| \leq \frac{3 r}{1-r^{2}} .
$$

As the classes we discuss here are all subclasses of starlike functions. By (2.35), we have

$$
\operatorname{Re} \frac{z f^{\prime}(z)}{f(z)} \geq \frac{1-3 r}{1-r^{2}} \geq 0, \quad(r \leq 1 / 3) .
$$

Hence all the radii that we estimate will be less than $1 / 3$. Note that, for $0<r \leq 1 / 3$, the centre of disk in 2.35) lies in the interval $[1,9 / 8] \approx[1,1.125]$. 
(i) The number $r=R_{\mathcal{S}^{*}(\alpha)}$ is the root of $\alpha r^{2}-3 r+1-\alpha=0$ in $[0,1]$ and hence, for $0<r \leq R_{\mathcal{S}^{*}(\alpha)}$, it follows by 2.36 that

$$
\operatorname{Re} \frac{z f^{\prime}(z)}{f(z)} \geq \frac{1-3 r}{1-r^{2}} \geq \alpha
$$

For the function $f_{3} \in \mathcal{F}_{3}$ given by 2.31 , we have

$$
\frac{z f_{3}^{\prime}(z)}{f_{3}(z)}=\frac{1-3 z}{1-z^{2}}=\frac{1-3 r}{1-r^{2}}=\alpha, \quad\left(z=r=R_{\mathcal{S}^{*}(\alpha)}\right)
$$

and this shows that the radius is sharp (See Figure 4.(a)).

(ii) It follows from 2.35 that

$$
\left|\frac{z f^{\prime}(z)}{f(z)}-1\right| \leq\left|\frac{z f^{\prime}(z)}{f(z)}-\frac{1}{1-r^{2}}\right|+\frac{r^{2}}{1-r^{2}} \leq \frac{3 r+r^{2}}{1-r^{2}}
$$

The number $r=R_{\mathcal{S}_{L}^{*}}$ is the root in $[0,1]$ of $\left(3 r+r^{2}\right)=(\sqrt{2}-1)\left(1-r^{2}\right)$ and for $0<r \leq R_{\mathcal{S}_{L}^{*}}$, we have

$$
\frac{3 r+r^{2}}{1-r^{2}} \leq \sqrt{2}-1
$$

Therefore, by 2.37) and 2.38), for $0<r \leq R_{\mathcal{S}_{L}^{*}}$, we have

$$
\left|\frac{z f^{\prime}(z)}{f(z)}-1\right| \leq \frac{3 r+r^{2}}{1-r^{2}} \leq \sqrt{2}-1
$$

For $0<r \leq R_{\mathcal{S}_{L}^{*}}$, using triangle inequality and 2.39 , we have

$$
\left|\frac{z f^{\prime}(z)}{f(z)}+1\right| \leq \sqrt{2}+1
$$

and hence by 2.39 and 2.40

$$
\left|\left(\frac{z f^{\prime}(z)}{f(z)}\right)^{2}-1\right| \leq\left|\frac{z f^{\prime}(z)}{f(z)}+1\right|\left|\frac{z f^{\prime}(z)}{f(z)}-1\right| \leq(\sqrt{2}+1)(\sqrt{2}-1)=1 .
$$

The number $\rho=R_{\mathcal{S}_{L}^{*}}$ satisfies $(1+3 \rho) /\left(1-\rho^{2}\right)=\sqrt{2}$. Using this, we see that the function $f_{3}$ defined in 2.31 satisfies

$$
\left|\left(\frac{z f_{3}^{\prime}(z)}{f_{3}(z)}\right)^{2}-1\right|=\left|\left(\frac{1-3 z}{1-z^{2}}\right)^{2}-1\right|=\left|\left(\frac{1+3 \rho}{1-\rho^{2}}\right)^{2}-1\right|=1, \quad\left(z:=-\rho=-R_{\mathcal{S}_{L}^{*}}\right) .
$$

This shows that the radius is sharp.

(iii) If $0<r \leq R_{\mathcal{S}_{p}}$, then $a=1 /\left(1-r^{2}\right) \leq 3 / 2$ and

$$
\frac{3 r}{1-r^{2}} \leq \frac{1}{1-r^{2}}-\frac{1}{2} \text {. }
$$

Thus, by 2.13), we see that the disk in 2.35) lies inside the parabolic region $\Omega_{P A R}$. Sharpness follows for the function $f_{3}$ defined in 2.31. At $z:=\rho=R_{\mathcal{S}_{p}}$, we have

$$
\operatorname{Re} \frac{z f^{\prime}(z)}{f(z)}=\frac{1-3 \rho}{1-\rho^{2}}=\frac{3 \rho-\rho^{2}}{1-\rho^{2}}=\left|\frac{\rho^{2}-3 \rho}{1-\rho^{2}}\right|=\left|\frac{z f^{\prime}(z)}{f(z)}-1\right| .
$$




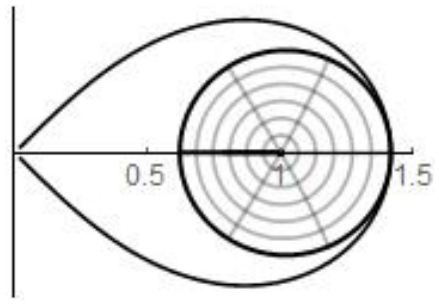

(A) Sharpness of class $\mathcal{S}_{L}^{*}$

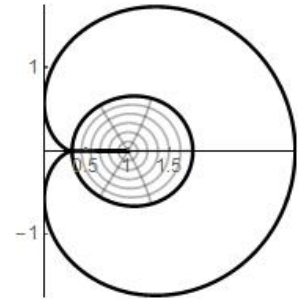

(B) Sharpness of class $\mathcal{S}_{c}^{*}$

Figure 4. Sharpness of starlike functions associated with lemniscate and cardioid.

(iv) For $0<r \leq R_{\mathcal{S}_{e}^{*}}$, we have $1 / e \leq a=1 /\left(1-r^{2}\right) \leq\left(e+e^{-1}\right) / 2$ and

$$
\frac{3 r}{1-r^{2}} \leq \frac{1}{1-r^{2}}-\frac{1}{e} \text {. }
$$

By (2.14), the disk in (2.35) lies inside $\Omega_{e}$ for $0<r \leq R_{\mathcal{S}_{e}^{*}}$ and it proves that the $\mathcal{S}_{e}^{*}$ radius for the class $\mathcal{F}_{3}$ is $R_{\mathcal{S}_{e}^{*}}$. The sharpness follows for the function $f_{3}$ defined in (2.31). Indeed at $z:=\rho=R_{\mathcal{S}_{e}^{*}}$, we have

$$
\left|\log \frac{z f_{1}^{\prime}(z)}{f_{1}(z)}\right|=\left|\log \frac{1-3 \rho}{1-\rho^{2}}\right|=1 .
$$

(v) If $0<r \leq R_{\mathcal{S}_{c}^{*}}$, then

$$
\frac{3 r}{1-r^{2}} \leq \frac{1}{1-r^{2}}-\frac{1}{3} .
$$

By (2.15), we see that the disk in (2.35) lies inside $\Omega_{c}$, if $0<r \leq R_{\mathcal{S}_{c}^{*}}$. The result is sharp for the function $f_{3}$ defined in (2.31) (See Figure 4. (b)). At $z:=\rho=R_{\mathcal{S}_{c}^{*}}$,

$$
\left|\frac{z f^{\prime}(z)}{f(z)}\right|=\left|\frac{1-3 \rho}{1-\rho^{2}}\right|=\frac{1}{3}=\Omega_{c}(-1) \in \partial \Omega_{c}(\mathbb{D}) .
$$

(vi) For $0<r \leq R_{\mathcal{S}_{s i n}^{*}}$

$$
\frac{3 r}{1-r^{2}} \leq \sin 1-\frac{r^{2}}{1-r^{2}} .
$$

It is evident from (2.16) that the disk in (2.35) lies inside $\Omega_{s}$. For the function $f_{3}$ defined in 2.31, at $z:=-\rho=-R_{\mathcal{S}_{s i n}^{*}}$,

$$
\left|\frac{z f^{\prime}(z)}{f(z)}\right|=\left|\frac{1+3 \rho}{1-\rho^{2}}\right|=1+\sin 1=q_{0}(1) \in \partial \Omega_{s}(\mathbb{D}) .
$$

(vii) If $0<r \leq R_{\mathcal{S}_{\mathbb{\zeta}}^{*}}$, then

$$
\frac{3 r-1}{1-r^{2}} \leq 1-\sqrt{2} .
$$

Thus, by (2.17), the disk in (2.35) lies inside $\left\{w \in \mathbb{C}:\left|w^{2}-1\right|<2|w|\right\}$ and hence $f \in \mathcal{S}_{\overparen{b}}^{*}$. The sharpness follows for the function defined in (2.31) (See Figure 5.(a)). 


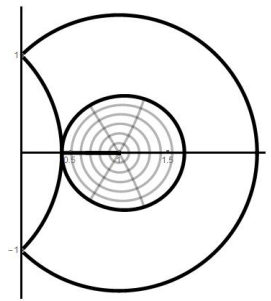

(A) Sharpness of class $\mathcal{S}_{\overparen{\zeta}}^{*}$

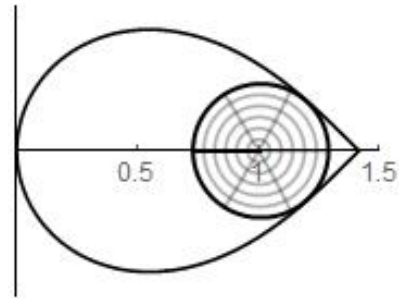

(B) Sharpness of class $\mathcal{S}_{R L}^{*}$

FiguRE 5. Sharpness of starlike functions associated with lune and reverse lemniscate.

At $z:=\rho=R_{\mathcal{S}_{\zeta}^{*}}$, we have

$$
\left|\left(\frac{z f_{3}^{\prime}(z)}{f_{3}(z)}\right)^{2}-1\right|=\left|\left(\frac{1-3 \rho}{1-\rho^{2}}\right)^{2}-1\right|=2\left|\frac{1-3 \rho}{1-\rho^{2}}\right|=2\left|\frac{z f_{3}^{\prime}(z)}{f_{3}(z)}\right| .
$$

(viii) If $0<r \leq R_{\mathcal{S}_{R}^{*}}, 2(\sqrt{2}-1)<a=1 /\left(1-r^{2}\right) \leq \sqrt{2}$ and

$$
\frac{3 r-1}{1-r^{2}} \leq 2-2 \sqrt{2}, \quad 0<r \leq R_{\mathcal{S}_{R}^{*}}
$$

Then, by 2.18), the disk in 2.35) lies inside $\psi(\mathbb{D})$. The result is sharp for the function defined in 2.31. At $z:=\rho=R_{\mathcal{S}_{R}^{*}}$,

$$
\left|\frac{z f^{\prime}(z)}{f(z)}\right|=\left|\frac{1-3 \rho}{1-\rho^{2}}\right|=2(\sqrt{2}-1)=\psi(1) \in \partial \psi(\mathbb{D}) .
$$

(ix) If $0<r \leq R_{\mathcal{S}_{R L}^{*}}, \sqrt{2} / 3 \leq a=1 /\left(1-r^{2}\right)<\sqrt{2}$, and

$$
\begin{aligned}
9 r^{2}-\left(1-r^{2}\right) & \sqrt{\left(1-r^{2}\right)^{2}-\left(\left(\sqrt{2}-\sqrt{2} r^{2}\right)-1\right)^{2}} \\
& +\left(1-r^{2}\right)^{2}-\left(\left(\sqrt{2}-\sqrt{2} r^{2}\right)-1\right)^{2} \leq 0 .
\end{aligned}
$$

Then, by (2.19), the disk in (2.35) lies inside the region $\left\{w:\left|(w-\sqrt{2})^{2}-1\right|<1\right\}$. The result is sharp for the function defined in (2.31) (See Figure 5.(b)).

(x) If $0<r \leq R_{\mathcal{S}_{\gamma}^{*}}, 0<\gamma \leq 1$, then $r \leq(\sin (\pi \gamma) / 2) / 3$. It is evident from (2.20) that the disk in 2.35) is contained in the sector $|\arg w| \leq(\pi \gamma) / 2,0<\gamma \leq 1$ if $0<r \leq R_{\mathcal{S}_{\gamma}^{*}}$

Definition 2.7. Let $\mathcal{F}_{4}$ be the class of functions $f \in \mathcal{A}$ satisfying the inequality

$$
\operatorname{Re}\left(\frac{(1+z)^{2}}{z} f(z)\right)>0 \quad(z \in \mathbb{D}) \text {. }
$$

The functions $f_{4}: \mathbb{D} \longrightarrow \mathbb{C}$ defined by

$$
f_{4}(z)=\frac{z(1-z)}{(1+z)^{3}}
$$

satisfy

$$
\operatorname{Re} \frac{(1+z)}{z} f_{4}(z)=\operatorname{Re} \frac{1-z}{1+z}>0
$$


and hence the function $f_{4} \in \mathcal{F}_{4}$. This proves that the class $\mathcal{F}_{4}$ is non-empty. Also, the function $f_{4}$ is extremal function for the radii problem we consider. Since

$$
f_{4}(z)=z-4 z^{2}+9 z^{3}-16 z^{4}+\ldots,
$$

the functions in $\mathcal{F}_{4}$ are not necessarily univalent. Since

$$
f_{4}^{\prime}(z)=\frac{1-4 z+z^{2}}{(1+z)^{4}},
$$

we have $f_{4}^{\prime}(2-\sqrt{3})=0$ and it follows by the first part of the following theorem, that the radius of univalence of the functions in class $\mathcal{F}_{4}$ is $2-\sqrt{3} \approx 0.276949$.

TheOREM 2.8. For the class $\mathcal{F}_{4}$, the following results hold:

(i) The $\mathcal{S}^{*}(\alpha)$ radius $R_{\mathcal{S}^{*}(\alpha)}=(1-\alpha) /\left(2+\sqrt{3+\alpha^{2}}\right), \quad 0 \leq \alpha<1$.

(ii) The $\mathcal{S}_{L}^{*}$ radius $R_{\mathcal{S}_{L}^{*}}=(\sqrt{5}-2) /(1+\sqrt{2}) \approx 0.9778$.

(iii) The $\mathcal{S}_{p}$ radius $R_{\mathcal{S}_{p}}=(4-\sqrt{13}) / 3 \approx 0.1315$.

(iv) The $\mathcal{S}_{e}^{*}$ radius $R_{\mathcal{S}_{e}^{*}}=(e-1) /\left(2 e+\sqrt{3 e^{2}+1}\right) \approx 0.1676$.

(v) The $\mathcal{S}_{c}^{*}$ radius $R_{\mathcal{S}_{c}^{*}}=(3-\sqrt{7}) / 2 \approx 0.1771$.

(vi) The $\mathcal{S}_{\text {sin }}^{*}$ radius $R_{\mathcal{S}_{\text {sin }}^{*}}=\sin 1 /(2+\sqrt{4+\sin 1(2+\sin 1)}) \approx 0.1858$.

(vii) The $\mathcal{S}_{\zeta}^{*}$ radius $R_{\mathcal{S}_{\zeta}^{*}}=\sqrt{2}-\sqrt{(3-\sqrt{2})} \approx 0.1549$.

(viii) The $\mathcal{S}_{R}^{*}$ radius $R_{\mathcal{S}_{R}^{*}}=(3-2 \sqrt{2}) /(2+\sqrt{15-8 \sqrt{2}}) \approx 0.0438$.

(ix) The $\mathcal{S}_{R L}^{*}$ radius $R_{\mathcal{S}_{R L}^{*}}$ is the root $(\approx 0.0694)$ in $[0,1]$ of the equation

$$
\begin{aligned}
16 r^{2}+ & \left(r^{2}-1\right)^{2}-\left(1-\sqrt{2}+(1+\sqrt{2}) r^{2}\right)^{2}+ \\
\left(r^{2}-1\right) \sqrt{2 \sqrt{2}-2-2(1+\sqrt{2}) r^{4}} & =0 .
\end{aligned}
$$

(x) The $\mathcal{S}_{\gamma}^{*}$ radius $R_{S_{\gamma}^{*}} \geq(\sin (\pi \gamma / 2)) /\left(2+\sqrt{4-\sin ^{2}(\pi \gamma / 2)}\right), \quad 0<\gamma \leq 1$.

Proof. Let the function $f \in \mathcal{F}_{4}$. Then

$$
\operatorname{Re}\left(\frac{(1+z)^{2}}{z} f(z)\right)>0 \quad(z \in \mathbb{D}) \text {. }
$$

Define the function $h: \mathbb{D} \longrightarrow \mathbb{C}$ by

$$
h(z)=\frac{(1+z)^{2}}{z} f(z) .
$$

By 2.42 and 2.43 we have $h \in \mathcal{P}$ and $f(z)=z h(z) /(1+z)^{2}$. Therefore, by calculation it can be shown that

$$
\frac{z f^{\prime}(z)}{f(z)}=\frac{z h^{\prime}(z)}{h(z)}+\frac{1-z}{1+z} .
$$

The bilinear transformation $(1-z) /(1+z)$ maps the disk $|z| \leq r$ onto the disk

$$
\left|\frac{1-z}{1+z}-\frac{1+r^{2}}{1-r^{2}}\right| \leq \frac{2 r}{1-r^{2}} .
$$


Using (2.45) and (2.6), it follows from (2.44) that $f$ maps the disk $|z| \leq r$ onto the disk

$$
\left|\frac{z f^{\prime}(z)}{f(z)}-\frac{1+r^{2}}{1-r^{2}}\right| \leq \frac{4 r}{1-r^{2}}
$$

The classes we discuss here are all subclasses of starlike functions. By (2.46), we have

$$
\operatorname{Re} \frac{z f^{\prime}(z)}{f(z)} \geq \frac{1-4 r+r^{2}}{1-r^{2}} \geq 0, \quad(r \leq 2-\sqrt{3}) .
$$

Hence all the radii that we estimate will be less than $2-\sqrt{3} \approx 0.26794$. Note that for $0<r \leq(2-\sqrt{3})$, the centre of disk in 2.46$)$ lies in the interval $[1,(4-2 \sqrt{3}) /(2 \sqrt{3}-3)] \approx$ $[1,1.1547]$.

(i) The number $r=R_{\mathcal{S}^{*}(\alpha)}$ is the root of $(1+\alpha) r^{2}-4 r+1-\alpha=0$ in $[0,1]$ and hence, for $0<r \leq R_{\mathcal{S}^{*}(\alpha)}$, it follows by 2.47 that

$$
\operatorname{Re} \frac{z f^{\prime}(z)}{f(z)} \geq \frac{1-4 r+r^{2}}{1-r^{2}} \geq \alpha .
$$

For the function $f_{4} \in \mathcal{F}_{4}$ given by $(2.41)$ we have

$$
\frac{z f_{4}^{\prime}(z)}{f_{4}(z)}=\frac{1-4 z+z^{2}}{1-z^{2}}=\frac{1-4 r+r^{2}}{1-r^{2}}=\alpha, \quad\left(z=r=R_{\mathcal{S}^{*}(\alpha)}\right)
$$

and this shows that the radius is sharp.

(ii) It follows from 2.46 that

$$
\left|\frac{z f^{\prime}(z)}{f(z)}-1\right| \leq\left|\frac{z f^{\prime}(z)}{f(z)}-\frac{1+r^{2}}{1-r^{2}}\right|+\frac{2 r^{2}}{1-r^{2}} \leq \frac{4 r+2 r^{2}}{1-r^{2}} .
$$

The number $r=R_{\mathcal{S}_{L}^{*}}$ is the root in $[0,1]$ of $\left(4 r+2 r^{2}\right) \leq(\sqrt{2}-1)\left(1-r^{2}\right)$ and for $0<r \leq R_{\mathcal{S}_{L}^{*}}$, we have

$$
\frac{4 r+2 r^{2}}{1-r^{2}} \leq \sqrt{2}-1
$$

Therefore, by 2.48 and 2.49 , for $0<r \leq R_{\mathcal{S}_{L}^{*}}$, we have

$$
\left|\frac{z f^{\prime}(z)}{f(z)}-1\right| \leq \frac{4 r+2 r^{2}}{1-r^{2}} \leq \sqrt{2}-1
$$

For $0<r \leq R_{\mathcal{S}_{L}^{*}}$, using triangle inequality and 2.50, we have

$$
\left|\frac{z f^{\prime}(z)}{f(z)}+1\right| \leq \sqrt{2}+1
$$

and hence by 2.50 and 2.51

$$
\left|\left(\frac{z f^{\prime}(z)}{f(z)}\right)^{2}-1\right| \leq\left|\frac{z f^{\prime}(z)}{f(z)}+1\right|\left|\frac{z f^{\prime}(z)}{f(z)}-1\right| \leq(\sqrt{2}+1)(\sqrt{2}-1)=1 .
$$

The number $\rho=R_{\mathcal{S}_{L}^{*}}$ satisfies $\left(1+4 \rho-\rho^{2}\right) /\left(1-\rho^{2}\right)=\sqrt{2}$. Using this, we see that at $z:=-\rho=-R_{\mathcal{S}_{L}^{*}}$, the function $f_{4}$ defined in 2.41 satisfies

$$
\left|\left(\frac{z f_{4}^{\prime}(z)}{f_{4}(z)}\right)^{2}-1\right|=\left|\left(\frac{1-4 z+z^{2}}{1-z^{2}}\right)^{2}-1\right|=\left|\left(\frac{1+4 \rho+\rho^{2}}{1-\rho^{2}}\right)^{2}-1\right|=1 \text {. }
$$




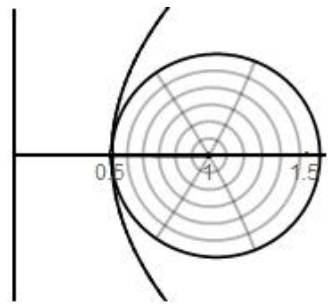

(A) Sharpness of class $\mathcal{S}_{p}$

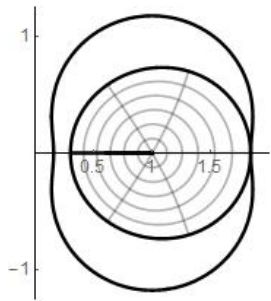

(B) Sharpness of class $\mathcal{S}_{\text {sin }}^{*}$

FiguRE 6. Sharpness of parabolic starlike functions and starlike functions associated with sine function

This shows that the radius is sharp.

(iii) If $0<r \leq R_{\mathcal{S}_{p}}$, then $a=1 /\left(1-r^{2}\right) \leq 3 / 2$ and

$$
\frac{4 r}{1-r^{2}} \leq \frac{1+r^{2}}{1-r^{2}}-\frac{1}{2} \text {. }
$$

Thus, by (2.13), we see that the disk in 2.46) lies inside the parabolic region $\Omega_{P A R}$. Sharpness follows for the function $f_{4}$ defined in (2.41) (See Figure 6. (a)). At $z:=\rho=R_{\mathcal{S}_{p}}$, we have

$$
\operatorname{Re} \frac{z f^{\prime}(z)}{f(z)}=\frac{1-4 \rho+\rho^{2}}{1-\rho^{2}}=\frac{4 \rho-2 \rho^{2}}{1-\rho^{2}}=\left|\frac{2 \rho^{2}-4 \rho}{1-\rho^{2}}\right|=\left|\frac{z f^{\prime}(z)}{f(z)}-1\right| .
$$

(iv) For $0<r \leq R_{\mathcal{S}_{e}^{*}}$, we have $1 / e \leq a=1 /\left(1-r^{2}\right) \leq\left(e+e^{-1}\right) / 2$ and

$$
\frac{4 r}{1-r^{2}} \leq \frac{1+r^{2}}{1-r^{2}}-\frac{1}{e}
$$

By (2.14), the disk in 2.46) lies inside $\Omega_{e}$ for $0<r \leq R_{\mathcal{S}_{e}^{*}}$ proving that the $\mathcal{S}_{e}^{*}$ radius for the class $\mathcal{F}_{4}$ is $R_{\mathcal{S}_{e}^{*}}$. The sharpness follows for the function $f_{4}$ defined in 2.41. Indeed at $z:=\rho=R_{\mathcal{S}_{e}^{*}}$, we have

$$
\left|\log \frac{z f_{4}^{\prime}(z)}{f_{4}(z)}\right|=\left|\log \frac{1-4 \rho+\rho^{2}}{1-\rho^{2}}\right|=1 .
$$

(v) If $0<r \leq R_{\mathcal{S}_{c}^{*}}$, then

$$
\frac{4 r}{1-r^{2}} \leq \frac{1+r^{2}}{1-r^{2}}-\frac{1}{3}
$$

By (2.15), we see that the disk in 2.46) lies inside $\Omega_{c}$, if $0<r \leq R_{\mathcal{S}_{c}^{*}}$. The result is sharp for the function $f_{4}$ defined in 2.41). At $z:=\rho=R_{\mathcal{S}_{c}^{*}}$,

$$
\left|\frac{z f^{\prime}(z)}{f(z)}\right|=\left|\frac{1-4 \rho+\rho^{2}}{1-\rho^{2}}\right|=\frac{1}{3}=\Omega_{c}(-1) \in \partial \Omega_{c}(\mathbb{D}) .
$$

(vi) For $0<r \leq R_{\mathcal{S}_{\text {sin }}^{*}}$

$$
\frac{4 r}{1-r^{2}} \leq \sin 1-\frac{2 r^{2}}{1-r^{2}}
$$




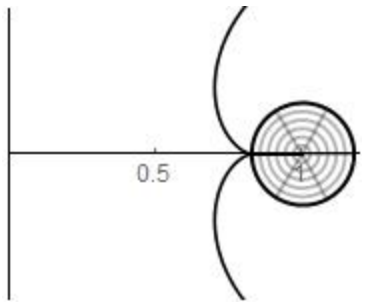

(A) Sharpness of class $\mathcal{S}_{R}^{*}$

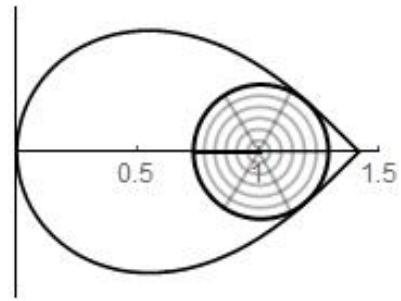

(B) Sharpness of class $\mathcal{S}_{R L}^{*}$

FIGURE 7. Sharpness of starlike functions associated with a rational function and reverse lemniscate.

It is evident from $(2.16)$ that the disk in 2.46$)$ lies inside $\Omega_{s}$ provided $0<r \leq R_{\mathcal{S}_{\text {sin }}^{*}}$.

For the function $f_{4}$ defined in 2.41) (See Figure 6.(b)), at $z:=-\rho=-R_{\mathcal{S}_{\text {sin }}^{*}}$,

$$
\left|\frac{z f^{\prime}(z)}{f(z)}\right|=\left|\frac{1+4 \rho+\rho^{2}}{1-\rho^{2}}\right|=1+\sin 1=q_{0}(1) \in \partial \Omega_{s}(\mathbb{D}) .
$$

(vii) If $0<r \leq R_{\mathcal{S}_{\zeta}^{*}}$, then

$$
\frac{4 r-1-r^{2}}{1-r^{2}} \leq 1-\sqrt{2}
$$

Thus, by (2.17), the disk in (2.46) lies inside $\left\{w \in \mathbb{C}:\left|w^{2}-1\right|<2|w|\right\}$ and hence $f \in \mathcal{S}_{\mathbb{\beta}}^{*}$. The sharpness follows from the function defined in (2.41). At $z:=\rho=R_{\mathcal{S}_{\zeta}^{*}}$, we have

$$
\left|\left(\frac{z f_{4}^{\prime}(z)}{f_{4}(z)}\right)^{2}-1\right|=\left|\left(\frac{\rho^{2}-4 \rho+1}{1-\rho^{2}}\right)^{2}-1\right|=2\left|\left(\frac{\rho^{2}-4 \rho+1}{1-\rho^{2}}\right)\right|=2\left|\left(\frac{z f_{4}^{\prime}(z)}{f_{4}(z)}\right)\right| \text {. }
$$

(viii) If $0<r \leq R_{\mathcal{S}_{R}^{*}}, 2(\sqrt{2}-1)<a=1 /\left(1-r^{2}\right) \leq \sqrt{2}$ and

$$
\frac{4 r-1-r^{2}}{1-r^{2}} \leq 2-2 \sqrt{2}, \quad 0<r \leq R_{\mathcal{S}_{R}^{*}}
$$

Then, by (2.18), the disk in (2.46) lies inside $\psi(\mathbb{D})$. The result is sharp for the function defined in 2.41) (See Figure 7.(a)). At $z:=\rho=R_{\mathcal{S}_{R}^{*}}$,

$$
\left|\frac{z f^{\prime}(z)}{f(z)}\right|=\left|\frac{1-4 \rho+\rho^{2}}{1-\rho^{2}}\right|=2(\sqrt{2}-1)=\psi(1) \in \partial \psi(\mathbb{D}) \text {. }
$$

(ix) If $0<r \leq R_{\mathcal{S}_{R L}^{*}}, \sqrt{2} / 3 \leq a=1 /\left(1-r^{2}\right)<\sqrt{2}$, and

$$
\begin{aligned}
16 r^{2}-\left(1-r^{2}\right) & \sqrt{\left(1-r^{2}\right)^{2}-\left(\left(\sqrt{2}-\sqrt{2} r^{2}\right)-\left(1+r^{2}\right)^{2}\right.} \\
& +\left(1-r^{2}\right)^{2}-\left(\left(\sqrt{2}-\sqrt{2} r^{2}\right)-\left(1+r^{2}\right)^{2} \leq 0 .\right.
\end{aligned}
$$

Then, by (2.19), the disk in (2.46) lies inside the region $\left\{w:\left|(w-\sqrt{2})^{2}-1\right|<1\right\}$. The result is sharp for the function defined in (2.41) (See Figure 7.(b)).

(x) If $0<r \leq R_{\mathcal{S}_{\gamma}^{*}}, 0<\gamma \leq 1$, then $\left.r^{2} \sin (\pi \gamma / 2)-4 r+\sin (\pi \gamma) / 2\right) \leq 0$. It is evident from (2.20) that the disk 2.46) is contained in the sector $|\arg w| \leq(\pi \gamma) / 2$, if $0<r \leq R_{\mathcal{S}_{\gamma}^{*}}$. 


\section{References}

[1] R. M. Ali, and V. Ravichandran, Uniformly convex and uniformly starlike functions, Ramanujan Mathematics Newsletter 21 (2011), no. 1, 16-30.

[2] R. M. Ali, N. E. Cho, N. K. Jain and V. Ravichandran, Radii of starlikeness and convexity for functions with fixed second coefficient defined by subordination, Filomat 26 (2012), no. 3, 553-561.

[3] R. M. Ali, N. K. Jain and V. Ravichandran, Radii of starlikeness associated with the lemniscate of Bernoulli and the left-half plane, Appl. Math. Comput. 218 (2012), no. 11, 6557-6565.

[4] R. M. Ali, N. K. Jain and V. Ravichandran, On the radius constants for classes of analytic functions, Bull. Malays. Math. Sci. Soc. (2) 36 (2013), no. 1, 23-38.

[5] M. K. Aouf, J. Dziok and J. Sokół, On a subclass of strongly starlike functions, Appl. Math. Lett. 24 (2011), no. 1, 27-32.

[6] N. E. Cho, S. Kumar, V. Kumar and V. Ravichandran, Differential subordination and radius estimates for starlike functions associated with the Booth lemniscate, Turkish J. Math. 42 (2018), no. 3, 13801399.

[7] N. E. Cho, V.Kumar, S. S. Kumar and V. Ravichandran, Radius problems for starlike functions associated with the sine function, Bull. Iranian Math. Soc. 45 (2019), no. 1, 213-232.

[8] P. L. Duren, Univalent Functions, GTM, 259, Springer-Verlag, New York, 1983.

[9] S. Gandhi and V. Ravichandran, Starlike functions associated with a lune, Asian-Eur. J. Math. 10 (2017), no. 4, 1750064, 12 pp.

[10] A. Gangadharan, V. Ravichandran and T. N. Shanmugam, Radii of convexity and strong starlikeness for some classes of analytic functions, J. Math. Anal. Appl. 211 (1997), no. 1, 301-313.

[11] A. W. Goodman, Univalent Functions. Vol. II, Mariner, Tampa, FL, 1983.

[12] W. Janowski, Extremal problems for a family of functions with positive real part and for some related families, Ann. Polon. Math. 23 (1970/1971), 159-177.

[13] W. Janowski, Some extremal problems for certain families of analytic functions. I, Ann. Polon. Math. 28 (1973), 297-326.

[14] M. F. Kocur, On a class of univalent functions in the circle, Uspehi Mat. Nauk 17 (1962), no. 4 (106), $153-156$.

[15] M. F. Kocur, Bounds of starlikeness and convexity for certain special classes of analytic functions in the disc, Izv. Vysš. Učebn. Zaved. Matematika 1977, no. 7 (182), 57-60.

[16] M. F. Kocur, Radii of starlikeness and of convexity in some classes of analytic functions in the disc, Mat. Zametki 25 (1979), no. 5, 675-679, 798.

[17] I. M. Kotsur and M. F. Kotsur, Ukrainian Math. J. 48 (1996), no. 7, 1079-1083 (1997); translated from Ukraïn. Mat. Zh. 48 (1996), no. 7, 954-957.

[18] I. M. Kotsur and M. F. Kotsur, Russian Math. (Iz. VUZ) 41 (1997), no. 10, 46-48 (1998); translated from Izv. Vyssh. Uchebn. Zaved. Mat. 1997, no. 10, 48-50.

[19] B. Kowalczyk and A. Lecko, Radius problem in classes of polynomial close-to-convex functions I, Bull. Soc. Sci. Lett. Łódź Sér. Rech. Déform. 63 (2013), no. 1, 65-77.

[20] B. Kowalczyk and A. Lecko, Radius problem in classes of polynomial close-to-convex functions II. Partial solutions, Bull. Soc. Sci. Lett. Łódź Sér. Rech. Déform. 63 (2013), no. 2, 23-34.

[21] B. Kowalczyk and A. Lecko, The Fekete-Szegö problem for close-to-convex functions with respect to the Koebe function, Acta Math. Sci. Ser. B (Engl. Ed.) 34 (2014), no. 5, 1571-1583.

[22] B. Kowalczyk, A. Lecko and B. Śmiarowska, On some coefficient inequality in the subclass of close-toconvex functions, Bull. Soc. Sci. Lett. Łódź Sér. Rech. Déform. 67 (2017), no. 1, 79-90.

[23] A. Lecko, Some subclasses of close-to-convex functions, Ann. Polon. Math. 58 (1993), no. 1, 53-64.

[24] A. Lecko and Y. J. Sim, Coefficient problems in the subclasses of close-to-star functions, Results Math. 74 (2019), no. 3, Art. 104, 14 pp.

[25] U. Pranav Kumar and A. Vasudevarao, Logarithmic coefficients for certain subclasses of close-to-convex functions, Monatsh. Math. 187 (2018), no. 3, 543-563.

[26] S. Kumar and V. Ravichandran, A subclass of starlike functions associated with a rational function, Southeast Asian Bull. Math. 40 (2016), no. 2, 199-212.

[27] S. Kumar, N. E. Cho, V. Ravichandran and H.M. Srivastava, Sharp coefficient bounds for starlike functions associated with the Bell numbers, Math. Slovaca (2019), accepted. 
[28] W. C. Ma and D. Minda, Uniformly convex functions, Ann. Polon. Math. 57 (1992), no. 2, 165-175.

[29] W. C. Ma and D. Minda, A unified treatment of some special classes of univalent functions, in Proceedings of the Conference on Complex Analysis (Tianjin, 1992), 157-169, Conf. Proc. Lecture Notes Anal., I, Int. Press, Cambridge, MA.

[30] T. H. MacGregor, The radius of convexity for starlike functions of order $\frac{1}{2}$, Proc. Amer. Math. Soc. 14 (1963), 71-76.

[31] T. H. MacGregor, The radius of univalence of certain analytic functions, Proc. Amer. Math. Soc. 14 (1963), 514-520.

[32] T. H. MacGregor, A class of univalent functions, Proc. Amer. Math. Soc. 15 (1964), 311-317.

[33] R. Mendiratta, S. Nagpal and V. Ravichandran, A subclass of starlike functions associated with left-half of the lemniscate of Bernoulli, Internat. J. Math. 25 (2014), no. 9, 1450090, 17 pp.

[34] R. Mendiratta, S. Nagpal and V. Ravichandran, On a subclass of strongly starlike functions associated with exponential function, Bull. Malays. Math. Sci. Soc. 38 (2015), no. 1, 365-386.

[35] E. Paprocki and J. Sokół, The extremal problems in some subclass of strongly starlike functions, Zeszyty Nauk. Politech. Rzeszowskiej Mat. No. 20 (1996), 89-94.

[36] R. K. Raina and J. Sokół, Some properties related to a certain class of starlike functions, C. R. Math. Acad. Sci. Paris 353 (2015), no. 11, 973-978.

[37] J. S. Ratti, The radius of univalence of certain analytic functions, Math. Z. 107 (1968), $241-248$.

[38] J. S. Ratti, The radius of convexity of certain analytic functions, Indian J. Pure Appl. Math. 1 (1970), no. $1,30-36$.

[39] V. Ravichandran, F. Rønning and T. N. Shanmugam, Radius of convexity and radius of starlikeness for some classes of analytic functions, Complex Variables Theory Appl. 33 (1997), no. 1-4, 265-280.

[40] F. Rønning, Uniformly convex functions and a corresponding class of starlike functions, Proc. Amer. Math. Soc. 118 (1993), no. 1, 189-196.

[41] G. M. Shah, On the univalence of some analytic functions, Pacific J. Math. 43 (1972), 239-250.

[42] T. N. Shanmugam, Convolution and differential subordination, Internat. J. Math. Math. Sci. 12 (1989), no. $2,333-340$.

[43] T. N. Shanmugam and V. Ravichandran, Certain properties of uniformly convex functions, in Computational Methods and Function Theory 1994 (Penang), 319-324, Ser. Approx. Decompos., 5, World Sci. Publ., River Edge, NJ.

[44] K. Sharma, N. K. Jain and V. Ravichandran, Starlike functions associated with a cardioid, Afr. Mat. 27 (2016), no. 5-6, 923-939.

[45] J. Sokół and J. Stankiewicz, Radius of convexity of some subclasses of strongly starlike functions, Zeszyty Nauk. Politech. Rzeszowskiej Mat. No. 19 (1996), 101-105.

Department of Mathematics, National Institute of Technology, Tiruchirappalli-620015, INDIA

E-mail address: ashanitt18@gmail.com

Department of Mathematics, National Institute of Technology, Tiruchirappalli-620015, INDIA

E-mail address: vravi68@gmail.com; ravic@nitt.edu 